\title{
Network Coding and Cross-Layer Approach for Reliability and Optimization of Routing in VANET: A Survey
}

\author{
Vandana Ratwani \\ Charusat University \\ Changa, Taluka: Petlad, Dist: Anand \\ Gujarat, India-388421
}

\author{
Arpita Shah \\ Charusat University \\ Changa, Taluka: Petlad, Dist: Anand \\ Gujarat, India-388421
}

\begin{abstract}
Vehicular Ad-hoc Networks (VANET) is a subclass of mobile ad hoc networks. VANET provides wireless communication among vehicles and vehicle to roadside equipment. The communication between vehicles is regarding safety, comfort and entertainment. The performance of whole system depends on the reliability of the system and the routing of messages. Routing depends on the kind of routing protocol that is used.

In this study we, aim to study the routing protocols designed specifically for VANET. Due to the highly dynamic nature and set of challenges in the VANET environment, routing techniques designed for MANET cannot work with VANET. In order to increase the reliability and optimization of routing protocols, researchers have suggested methods like Network Coding and cross layer approach. This paper also gives an overview of these and effectiveness of these methods.
\end{abstract}

\section{General Terms}

Wireless Ad-hoc Networks, Vehicular Ad-hoc Networks (VANET), Routing protocols.

\section{Keywords}

VANET, Routing in VANET, Network Coding, Cross-Layer approach, Optimization.

\section{INTRODUCTION}

AN Ad-hoc network typically means any set of network where all devices have equal status on a network and are free to associate with all the other devices in the link range of network. Ad hoc network often refers to a mode of operation of IEEE 802.11 wireless networks. It also refers to a networks device's capability to record link status information for any number of devices in a 1-link range, and so it is often a layer 2 activity. Because this is a only a layer 2 activity, Ad-hoc networks alone may not support a routable IP network environment without additional layer 2 or layer 3 capabilities.

Vehicular Ad-hoc Networks (VANET) is a subclass of mobile Ad-hoc networks. VANET assists vehicle drivers to communicate and co-ordinate among themselves in order to avoid any critical situation through $\mathrm{V} 2 \mathrm{~V}$ communication via means of exchanging messages.

These messages can be about traffic jams, accident prone zones, speed limits passage of emergency and unseen obstacles. The most well-known application of these kind of systems include, "Advance Driver Assistance systems (ADASE2) Crash avoidance Matrices Partnership (CAMP), CARTALK2000 and fleet NET VanetMobsim."

Applications of VANET include:

To increase safety

Traffic optimization
To create Intelligent Transportation Systems

\section{ROUTING IN VANET}

The task of routing in VANET is highly challenging, due to unique characteristics of VANET like dynamic topology, frequent disconnections in the network, mobility modelling, communication environment, delay constraints and location identification. Many routing protocols have been proposed for VANET. These protocols are classified under position based routing techniques, broadcast routing techniques, hierarchical/cluster based routing techniques, geo-cast routing techniques and adaptive and context aware routing techniques. Literature shows that routing protocols that work well with MANET do not work well with VANET due its unique characteristics. [3] Thus Routing techniques that take into account VANET characteristics are required. Also the mobility model in VANET is different for highways and for city scenarios. Different protocols or protocols with variation are proposed for highway and city networks. The road based protocols in VANET are classified in to three types 1) Rural 2) Highway 3) Urban. All these three types encounter different kind of challenges. Rural area based protocols frequently encounter connectivity issues because of low network density. Urban routing protocols face challenges of bidirectional node movement and, in addition to angular node movements as in street map, thus reducing correlation between destination and next suitable hop. With highway protocols the high speed to node poses the problem, but in spite of this, the communication in highway is good, since all the nodes communicate in one direction. [1]

VANET routing protocols need to take under consideration, the nature of node movements, vehicle mobility, which is higher, direction of vehicle and information movement. Since wireless channels have unique problems like limited bandwidth, varying channel capacity and location-dependent channel errors the end-to-end delay becomes another issue of concern.

Routing in VANET has been studied recently and many protocols are proposed. These protocols are classified under three categories

Unicast Approach

Multicast and Geo-cast Approach

Broadcast Approach

Table 1. Comparison of VANET routing protocols

\begin{tabular}{|c|c|c|c|}
\hline Protocols & $\begin{array}{l}\text { Routing } \\
\text { Mechanism }\end{array}$ & $\begin{array}{l}\text { Forwarding } \\
\text { Strategy }\end{array}$ & $\begin{array}{c}\text { Recovery } \\
\text { strategy }\end{array}$ \\
\hline AODV & Unicast & Multi-hop & $\begin{array}{c}\text { Store \& } \\
\text { forwarding }\end{array}$ \\
\hline
\end{tabular}




\begin{tabular}{|c|c|c|c|}
\hline GPRS & Unicast & Greedy & $\begin{array}{c}\text { Store \& } \\
\text { forwarding }\end{array}$ \\
\hline VADD & Unicast & Greedy & $\begin{array}{c}\text { Store \& } \\
\text { forwarding }\end{array}$ \\
\hline DV-CAST & Broadcast & Multi-hop & $\begin{array}{c}\text { Store \& } \\
\text { forwarding }\end{array}$ \\
\hline GSR & Broadcast & Greedy & $\begin{array}{c}\text { Store \& } \\
\text { forwarding }\end{array}$ \\
\hline DSDV & Broadcast & Multi-hop & $\begin{array}{c}\text { Store \& } \\
\text { forwarding }\end{array}$ \\
\hline ZRP & Multicast & Greedy & Flooding \\
\hline
\end{tabular}

\section{NETWORK CODING}

Networking coding is a new research area that has found prospects in increasing reliability of existing network systems. Basically there are two main benefits of this approach: potential throughput improvements and a high degree of robustness.

In this approach instead of simply forwarding packets to next hop, nodes can recombine more than one input packets into one or several output packets. With this technique being used, the successful receipt of desired data will not depend on which packets are being received, instead it will depend on receiving sufficient number of individual packets. [2]

Coders replace routes and switches in network coding. Instead of directly forwarding the packets to their ultimate destination, the coders transmit metadata in the form of digital evidence about the message along multiple paths. Two or more metadata are combined into a single packet at the receiving end. This distribution scheme, by minimizing the number and severity of bottlenecks can help in increasing the effective capacity of the network.

Benefits of network coding include:

1. Efficient Bandwidth Usage

2. Increase in throughput

3. Improved fault tolerance

4. Increased reliability

\section{Mathematical Proof for Network coding}

$$
\begin{array}{r}
M=\left[\begin{array}{l}
1 \\
2
\end{array}\right] G=\left(\begin{array}{ll}
1 & 2 \\
3 & 4
\end{array}\right) E=\left[\begin{array}{l}
1 \\
2
\end{array}\right]\left(\begin{array}{ll}
1 & 2 \\
3 & 4
\end{array}\right)=\left[\begin{array}{l}
1.1+2.2 \\
1.3+2.4
\end{array}\right] \\
=\left[\begin{array}{l}
1+4 \\
3+8
\end{array}\right]=\left[\begin{array}{c}
5 \\
11
\end{array}\right] \quad E=\left[\begin{array}{c}
5 \\
11
\end{array}\right] \text { and }
\end{array}
$$

$G=\left(\begin{array}{ll}1 & 2 \\ 3 & 4\end{array}\right)$, generated is $G^{\prime}=\left(\begin{array}{ll}5 & 6 \\ 7 & 0\end{array}\right)$

$E^{\prime}=\left[\begin{array}{c}5 \\ 11\end{array}\right]\left(\begin{array}{ll}5 & 6 \\ 7 & 0\end{array}\right)=\left[\begin{array}{l}5.5+11.6 \\ 5.7+11.0\end{array}\right]=\left[\begin{array}{c}25+66 \\ 35+0\end{array}\right]=\left[\begin{array}{c}91 \\ 35\end{array}\right]$

$k=G^{\prime}, \quad G=\left(\begin{array}{ll}5 & 6 \\ 7 & 0\end{array}\right)\left(\begin{array}{ll}1 & 2 \\ 3 & 4\end{array}\right)=\left(\begin{array}{cc}5+18 & 10+24 \\ 7+0 & 14+0\end{array}\right)$ $=\left(\begin{array}{cc}23 & 34 \\ 7 & 14\end{array}\right)$

$E^{\prime}=\left[\begin{array}{l}91 \\ 35\end{array}\right] k$

$=\left(\begin{array}{cc}23 & 34 \\ 7 & 14\end{array}\right)$ System of 2 linear eqautions, $k M$

$=E^{\prime}$, so $E^{\prime}$ inverse $(k)$ $\operatorname{inverse}(k)=1 / 84\left(\begin{array}{cc}14 & -34 \\ -7 & 23\end{array}\right)=\left(\begin{array}{cc}14 / 84 & -34 / 84 \\ -7 / 84 & 23 / 84\end{array}\right)$
Solution $M=\left[\begin{array}{l}91 \\ 35\end{array}\right]\left(\begin{array}{cc}14 / 84 & -34 / 84 \\ -7 / 84 & 23 / 84\end{array}\right)=\left[\begin{array}{l}1 \\ 2\end{array}\right]$

Applications of Network Coding

1. $\mathrm{P} 2 \mathrm{P}$ file distribution

2. Wireless Networks

3. Ad-hoc Sensor Networks

4. Network Tomography

5. Network Security

\section{CROSS-LAYER APPROACH}

Different layers interact with each other by the means of standard interfaces provided in layered architectures. This can affect the performance of the network as whole, resulting in performance degradation. For instance the loss of packet due to channel environments and interference can be interpreted by different layers in different ways. Network layer might start node discovery process thinking the loss is due to node is out of reach. Transport layer might find it to be case excessive congestion. Whereas Medium Access Control (MAC) may interpret it as a result of collisions. All this leads to le Optimization plays a key role, while evaluating any network's efficiency. Likewise, in order to optimize the performance of VANET, many researchers have proposed architecture based on cross layer approach. Such cross-layer based designs enable interaction among two or more layers and encourages joint decision making. This section surveys the proposals based on cross-layer design approach. [3]

\subsection{MAC Layer- Network Layer}

In this type of cross layer integration MAC layer and network layer are included. These layers interact with each other to take optimized routing decisions. In this type of approach, the RSSI (Received signal Strength Indicator) information will be retrieved from MAC layer. This information will then be passed on the network layer for taking routing decisions. Network layer will send packets only to those nodes which are having less RSSI value. Less RSSI value indicates that the nodes are running at a high speed towards the destination node. Forwarding packets to such intermediate nodes will eventually make it reach faster to destination node. The whole network performance can be improved by selecting most appropriate relaying node

This will result in low latency in data delivery and will reduce network overhead by sending data to only nodes with less RSSI value in radio range.

\subsection{Layer Triggering}

In layer triggering, when one layer detects any issue, it immediately notifies other layers about it using a predefined signal. To lessen the problem of network congestion in DSRC networks, authors in [4] proposed adaptive congestion control scheme. Information is exchanged between MAC and application layer to control the rate at which application traffic is generated. The message generation rate is controlled at application layer by sending a congestion signal.

\section{NETWORK SIMULATORS}

A lot of simulators for VANET have emerged in recent years. Three types of simulators are developed in VANET. 


\section{Network simulators \\ 3. Integrated simulators}

\subsection{Mobility simulators}

The IMPORTANT framework has been one of the first attempt to understand the dependence between vehicular traffic and communication performance. However, the code is implemented in $\mathrm{C}$ by the author. Which makes it difficult to extend without support of a detailed documentation and debug.

In [6], VANETMobiSim simulator, written in java is presented, which can generate mobility traces. It is an open source vehicular mobility generator tool based on CanuMobisim. Another powerful mobility simulator, which makes use of a cellular automaton for simulating the interaction of vehicles is TranSim.Also SUMO is another simulator intended for traffic planning and road design optimization. [5]

\subsection{Network Simulators}

NS-2 supports both wired and wireless networks and is an open-source discrete event network, which also includes MANET routing protocols and implementation of IEEE 802.11 MAC layer. This simulator is most widely for academic networking research. Users interact with NS-2 by writing TCL scripts. The core OS NS-2 is written in C++.

OPNET is a commercial network simulator used for simulations of both wired and wireless networks.

OMNeT++ is an object oriented modular discrete event network simulator. OMNet++ has a Mobility Framework and INET Framework modules that provide support to network and mobility models. In OMNeT++ protocols can be supported through modules. [5]

\subsection{Integrated Simulators}

Integrated simulators are combination of two sub simulators: mobility simulator and network simulator, which communicate with each other. High level of maturity is provided ye both the simulators. GrooveNet is an integrated simulator implemented in C++ and Qt graphics cross-platform library in Linux. SWANS++ extends the network simulator SWANS by providing a GUI for visualization of scenario and mobility model. SUMO is paired with OMNeT++ by extending SUMO to allow it to communicate through TCP connection to OMNeT++. [5]

The main properties of VANET simulators are as follows.

1. The simulator should be open source for availability to others in order to accept or criticise the model.

2. For other to validate the model, the code should be clear

3. In order to analyse the content, the structure of the code should be modular

4. To achieve realistic vehicular traffic simulations, the mobility model has to take into account both macromobility and micro-mobility features of the scenarios.

5. High level of maturity is required in case of integrated simulators.

\section{CONCLUSION}

VANET is an emerging research field. Due to its unique characteristics and dynamic nature, developing an efficient routing protocol for VANET, which can cater to its changing topology at every time and provide higher reliability is challenging. Also literature shows that MANET routing protocols do not perform well with VANET characteristics. We need different routing protocols to serve diffident VANET applications and scenarios. A majority of work in the past has been done on development of routing techniques based on layered architecture without taking into consideration, channel conditions and performance limitations of lower layers, resulting in restricted performance. The paper provides the discussion on the cross layer design based architectures. Also it highlights the significance of the use of network coding for increasing the reliability of routing protocols. Both these techniques when implemented, will increase the overall performance and will play a key role in bringing VANET to the reality.

\section{REFERENCES}

[1] P.S Nithya Darisini and N. Santhiya Kumari "A Survey of Routing Protocols for VANET in Urban Scenarios", IEEE International conference on Pattern Recognition, Informatics and Mobile Engineering February 2013.

[2] Christina Fragouli, Jean-Yves Le Boudec and Jorg Widmer "Network Coding: An Instant Primer".

[3] Harsh trivedi, Prakash Veeraraghavan, Seng Loke, Aniruddh desai and Jack Singh, "Routing Mechanism and Cross-Layer Design for Vehicular Ad Hoc Networks: A Survey" IEEE Symposium on computer \& Informarics 2011.

[4] H.Jianhua, et al., "Adaptive Congestion control for DSRC vehicle networks", in IEEE coomun. Letters, vol. 14, pp. 127-129, 2010.

[5] Evjola Spaho*, Leonard Barolli, Gjergli Mino, Fatos Xhafa and Vladi Kolici "VANET Simulators: A Survey on Mobility and Routing Protocols, IEEE International conference on Broadband and Wireless Computing, Communication and application 2011.

[6] M.Fiore, J. Harri, F.Filali, and C. Bonnet, Vehicular Mobility simulation for VANETs, proc. Of the 40-th annual Simulation symposium (ANSS-2007), pp. 301309, 2007.

[7] Ning Wang, Kin Hon Ho, George Pavlou and Michael Howarth, University of Surrey, "An Overview of Routing Optimization for Internet Traffic Engineering" in Volume 1 IEEE, 2008.

[8] Jiayue He and Jennifer Rexford, "Toward Internet-Wide Multipath Routing", in IEEE Network, March/April 2008.

[9] Jiayue He, Jennifer Rexford, Mung Chiang,: Don't Optimize Existing Protocols, Design Optimizable Protocols" in News Letter, ACM SIGCOMM Computer Communication, Volume 37 Issue 3, July 2007.

[10] Jiayue He, Mung Chiang, Jennifer Rexford, "Can Congestion Control and Traffic Engineering $\mathrm{Be}$ at Odds?" GLOBECOM 2006.

[11] C. Fragouli and E. Soljanin, I"network coding applications"," tech. rep., Foundations and Trends in Networking Vol. No.2, 2007. 
[12] Amit N. Thakare, Mrs. M. Y. Joshi, "Performance Analysis of AODV \& DSR Routing Protocol in Mobile Ad hoc Networks", IJCA Special Issue on "Mobile Adhoc Networks", MANETs, 2010.

[13] Z. Narmawala and S. Srivastava, I"survey of applications of network coding in wired and wireless networks"," tech. rep., in Proceedings of the 14th National Conference on Communications, pp. 153-157,2008.

[14] Sherali Zeadally, Ray Hunt, Yuh-Shyan Chen ,Angela Irwin \& Aamir Hassan, "Vehicular ad hoc networks (VANETS): status, results, and challenges", Springer
Science+Business Media, LLC 2010 .

[15] Jiayue He, Ma'ayan Bresler,Mung Chiang, Jennifer Rexford, " Towards Robust Multi-Layer Traffic Engineering: Optimization of Congestion Control and Routing", IEEE JOURNAL ON SELECTED AREAS IN COMMUNICATIONS, VOL. 25, NO. 5, JUNE 2007.

[16] Moez Jerbi, Sidi-Mohammed Senouci, Rabah Meraihi and Yacine Ghamri-Doudane France Telecom R\&D, Core Network Laboratories, Lannion, France "An Improved Vehicular Ad Hoc Routing Protocol for City Environments" 2007. 